\title{
La plataforma de apoyo a la docencia como opción metodológica para el aprendizaje de competencias
}

\author{
The Support Platform for Educators as a \\ Methodological Option for Competitive Learning
}

\author{
Alejandro Salicetti Fonseca \\ Profesor de la Escuela de Educación Física y Deportes \\ Universidad de Costa Rica \\ San José, Costa Rica \\ alejandro.salicetti@ucr.ac.cr \\ Cipriano Romero Cerezo \\ Profesor de la Universidad de Granada \\ Granada, España \\ cromero@ugr.es
}

Recibido 29-01-2010 • Aceptado 28-03-2010 • Corregido 22-04-2010

\begin{abstract}
Resumen: El estudio tiene como objetivo comprobar el efecto sobre la aplicación de la Plataforma de Apoyo a la Docencia (SWAD) como metodología para el aprendizaje de competencias. Participaron dos grupos: $G E$ (n: 111) y $G V(n: 114)$, ambos de estudiantes de la Universidad de Granada (España). Se utilizó un cuestionario con preguntas cerradas, validado específicamente para el estudio y con alta fiabilidad. Se obtuvieron datos cualitativos mediante el portfolio, construido durante el curso como herramienta para el aprendizaje de competencias profesionales. El análisis descriptivo, tomando en consideración que los rangos de puntuación van de 1 a 5, presenta expectativas $y$ valoraciones altas, 4.13 (0.88); y el análisis de contraste muestra diferencias significativas $(p \leq 0.05)$ entre las expectativas iniciales y las valoraciones finales en todos los items del cuestionario, lo que indica una buena opinión por parte de los estudiantes ante este tipo de metodología y fortalece la idea de que este tipo de estrategia metodológica es válida para el desarrollo de competencias en la formación profesional. Los resultados cualitativos destacan
\end{abstract}

\section{Introducción}

Se muestra en la siguiente investigación una experiencia para lograr un ajuste a los nuevos enfoques del Espacio Europeo de Educación Superior (EEES) mediante el crédito ECTS (European Credit Transfer and Accumulation System). Para lograrlo, se partió de diversas disposiciones metodológicas que se desarrollan en distintas instituciones y comisiones de coordinación universitaria con el propósito fundamental de mejorar la calidad de la docencia, dentro de la Titulación de Maestro Especialista en Educación Física (EF) y como parte del proyecto de Innovación Docente de la Universidad de Granada, que a su vez está integrado en 
que la metodológica ha favorecido la capacidad de comunicarse de forma escrita y la utilización de ordenadores, mejorando la capacidad de trabajo individual mediante aprendizajes activos $y$ desarrollando la capacidad de análisis y síntesis de documentos.

Palabras clave: Metodologías docentes, Plataforma de Apoyo a la Docencia, proceso de aprendizaje, Educación Física.

Abstract: The study's objective is to test the effect of implementation on the Support Platform for Educators (SWAD) as a method for competitive learning. Two groups participated: $G E$ (n: 111) and $G V$ (n: 114), both groups of students were from the University of Granada (Spain). The questionnaire used closed questions, validated specifically for the study with high reliability. In addition, qualitative data was obtained through the portfolio, constructed during the course as a tool for competitive professional learning. The descriptive analysis took into consideration a range from 1 to 5 , and presented expectations and high assessments, 4.13 (0.88), and the analysis, by contrast, demonstrated significant differences $(p \leq 0.05)$ between initial expectations and final assessments in all items of the questionnaire, which indicated a good view of students involved in this type of methodology, and strengthened the idea that this type of methodological strategy is valid for the development of competitive professional training. The qualitative results highlighted that the methodology favored communication abilities in written form and the use of computers, improving individual work capacity through active learning and developing a capacity for document analysis and synthesis.

Key words: Teaching Methodologies, Support Platform for Teaching, Learning Process, Physical Education. un Proyecto de Excelencia de la Junta de Andalucía (Bolivar, 2006). Igualmente, hemos pretendido combinar la conexión entre la docencia y la investigación para obtener evidencias de los nuevos enfoques y su contribución a la mejora de la enseñanza universitaria (González y Wagenaar, 2003; Shulman, 2004 y Centro de Innovación Docente para las Universidades Andaluzas [CIDUA], 2005).

El Ministerio de Educación y Ciencia en España [MEC] (2006), por medio del Consejo de Coordinación Universitaria, efectúa unas recomendaciones sobre lo que implica un nuevo estilo de trabajo del profesorado, fomentar las técnicas de información y comunicación en la docencia, promover el trabajo colaborativo, organizar la enseñanza en función de las competencias que se deban adquirir y potenciar la adquisición de herramientas de aprendizaje autónomo y permanente. En la situación actual en la que se encuentra la Educación Superior, es muy notable y continuo el debate sobre las competencias, que relacionan directamente la cultura universitaria con el aprendizaje a lo largo de la vida y el mundo del trabajo (Esteban y Sáez, 2008). Mediante el crédito ECTS la docencia debe estar centrada en el estudiante, lo que requiere propiciar los itinerarios de aprendizaje y las condiciones adecuadas para que los futuros profesionales desarrollen las competencias y encuentren las herramientas adecuadas para ello (Romero y Salicetti, 2009).

\section{Marco teórico}

\section{La Plataforma de Apoyo a la Docencia}

Actualmente, son muchas las experiencias educativas con redes informáticas y principalmente estas se deben al tremendo impacto social de Internet, por lo que existe un número muy alto de 
educadores que han decidido utilizar el fácil acceso a los ordenadores para incorporarlos no sólo en su práctica docente, sino también en desarrollo profesional (MEC, 2006).

Se considera la utilización de portales web de apoyo al aprendizaje, a la docencia y a la gestión de datos de los alumnos de una universidad como un aspecto importante a tomar en cuenta dentro de las metodologías aplicadas a los estudiantes. Tal y como mencionan Cañas, Calandria, Ortigosa, Ros y Díaz, (2007), facilita al profesor la realización de algunas tareas docentes y de gestión, como la difusión de documentos y la consulta de datos de los alumnos, pero sobre todo porque propicia la autonomía y el auto-aprendizaje del estudiante, ya que este puede hacer uso de tales servicios en cualquier instante y desde cualquier sitio (SWAD, Sistema Web de Apoyo a la Docencia de la Universidad de Granada).

La utilización de la plataforma SWAD como estrategia metodológica se presenta como una herramienta con diversas funcionalidades para alcanzar un mejor proceso de enseñanza-aprendizaje. Cañas et al. (2007) resumen las funcionalidades de esta herramienta tomando en cuenta los siguientes parámetros: administración de titulaciones y asignaturas, información y documentación en los diferentes cursos, evaluación del estudiantado, información y administración del cuerpo docente y el alumnado, comunicación entre usuarios y estadísticas y control de visitas.

Conociendo las limitaciones que mantienen las enseñanzas de tipo tradicional y con el propósito de aprovechar las supuestas ventajas que puede ofrecer la integración de estos recursos tecnológicos en las metodologías activas, se decidió su aplicación al curso de Educación Física y su Didáctica I en la facultad de Educación de la Universidad de Granada (España), porque representa nuevas posibilidades en la comunicación, colaboración y distribución de conocimientos. No es sólo un recurso de información en el cual la Web de la asignatura es un medio en ese proceso
(Benito y Cruz, 2005), sino también un recurso de aprendizaje constructivista y colaborativo, puesto que no es raro encontrar tutorías a distancia, por correo electrónico, grupos de discusión formados por el alumnado y el personal docente, servidores con materiales para estudio, listas de recursos de interés para la materia, obras de consulta en línea y otras muchas opciones que le permiten al estudiante utilizar diferentes herramientas para guiar y construir su propio aprendizaje.

Uno de los motivos que impulsaron la aplicación de diferentes estrategias metodológicas fue la necesidad del profesor de la asignatura de utilizar un espacio en el que pudiera acumular las experiencias que se van produciendo durante el desarrollo del curso. Y, además, que estas experiencias fueran accesibles para los demás estudiantes. El docente necesitaba herramientas nuevas para formarse, compartir sus experiencias, debatir problemas y buscar soluciones que agilizaran y facilitaran su labor. Para Sánchez (2007), estas herramientas deberían facilitar el paso de un trabajo individual, del conocimiento enclaustrado, al trabajo colaborativo y las comunidades de aprendizaje, modelo que se ajusta más a la nueva realidad social. El E-Learning se presenta como una respuesta de las Tecnologías de la Información y la Comunicación (TIC) para la innovación en la formación del profesorado, por ser una fórmula que pone en práctica un modelo de formación on-line que se dirige hacia el fomento de la participación del alumno como medio fundamental del aprendizaje, y en el que el profesorado ejerce el rol de guía de conocimientos (Gallego, 2003).

Los distintos servicios ofrecidos por Internet (web, FTP, blog, etc.) se han configurado en los últimos años como una de las opciones preferidas de apoyo a la educación, dando lugar a conceptos muy utilizados y estudiados en la actualidad como E-learning, campus 
virtuales, educación basada en la web, formación on-line, formación virtual, etc. (Fernández, Mir y Pablo-Martí, 2007). La Universidad de Granada, al igual que otras muchas universidades, dispone de estas herramientas para facilitar el aprendizaje en los alumnos.

Debido a la gran amplitud de opciones que presentan los aspectos antes mencionados, la estrategia metodológica que se ha aplicado para este trabajo de investigación está referida al papel de Internet en la formación y socialización profesional mediante el uso de la Plataforma de Apoyo a la Docencia, en donde, según Gallego (2003), esta integración de Internet en las aulas se apoya en un modelo híbrido de intervención que combina actividades virtuales y presenciales.

Para la utilización de las plataformas de apoyo a la docencia como estrategia metodológica, se aprovecha la oportunidad que se les presenta a los estudiantes de la titulación de Maestro Especialista en Educación Física de la Universidad de Granada de utilizar la plataforma SWAD. Es una plataforma de tele-formación desarrollada y utilizada en la Universidad en los últimos nueve cursos académicos, que de acuerdo con Cañas, Ortigosa y Aragón (2005) ofrece los servicios de información de las asignaturas, datos de estudiantes y profesores y comunicación entre usuarios.

Para lograr la aplicación de la metodología de la Plataforma de Apoyo a la Docencia fue preciso extender a los estudiantes del curso la oferta de servicios proporcionados a través de las tecnologías (plataforma SWAD), procesos de tutoría y asesoramiento agrupados en tutoría electrónica (virtual) y foro de discusión, comunicación simultánea y en tiempo real a través de chat, videoconferencias, pizarra compartida y otros sistemas interactivos de comunicación síncrona de indudable importancia en entornos de comunicación mediada por ordenador (Gallego, 2003).
Sobre la creación y puesta en funcionamiento de la plataforma SWAD, se puede decir que esta cumple con las condiciones necesarias que, según Fernández et al. (2007), deben darse para que este tipo de proyecto tenga el éxito deseado. Estas son:

- Apoyo institucional a iniciativas de este tipo.

- Liderazgo institucional del proyecto, aunando distintas iniciativas y dotando en su caso de los recursos necesarios para llevarlos a cabo.

- Identificación de un conjunto de materias apropiadas para iniciar una experiencia de este tipo.

- La buena disposición de grupos de profesores dispuestos a trabajar durante al menos un curso académico probando distintos modelos, metodologías y herramientas.

- Recursos adicionales necesarios. El soporte administrativo, el soporte técnico (plataforma, hardware, backups [respaldos], comunicaciones, etc.), la preparación de material didáctico y los recursos bibliográficos.

- Integración adecuada con las infraestructuras y las Tecnologías de Información y Comunicación (TIC) existentes.

La utilización de la Plataforma de Apoyo a la Docencia es una nueva asistencia a la enseñanza presencial, diferente a las clases tradicionales en donde el docente se encarga por completo de estructurar y organizar el total de las actividades del grupo. La utilización de la plataforma SWAD como fórmula más innovadora no implica hacer lo mismo que en las metodologías tradicionales; si el alumno utiliza el ordenador para realizar las tareas que antes escribía sobre un cuaderno, no se está en realidad ante un cambio del modelo propiciado con la ayuda de las TIC (Barquín, 2004). Por tal motivo, se quiere mostrar la importancia que puede tener 
el uso de la plataforma en el desarrollo competencial. Por ejemplo, la flexibilidad de horario para el uso de la plataforma y la disposición permanente permite acceder a ella en todo momento. Además, se dispone de una guía docente de la asignatura, que permite clarificar el camino a seguir por parte del estudiante en la adquisición de su aprendizaje. De igual manera, posibilita una comunicación entre el profesor y los estudiantes acerca de las dudas que estos puedan tener en cualquier momento, posibilitando la realización de tutorías virtuales (Gallego, 2003). Por último, mediante la estrategia metodológica de la plataforma SWAD de apoyo a la docencia, se les ha ofrecido a los estudiantes una amplia documentación sobre los trabajos efectuados por otros estudiantes, presentaciones empleadas en clase por el profesor y los mismos estudiantes, documentos de lectura previa a las clases, así como las referencias de la bibliografía necesaria para el estudio.

\section{Los docentes de Educación Física y sus competencias profesionales}

Los países de la Unión Europea viven en la actualidad políticas de convergencia que afectan a los planes de formación de profesionales; esto se nota principalmente en el ámbito de la Educación Superior y, como consecuencia, el diseño institucional de la oferta de formación y de capacitación docente está siendo sometido a un importante proceso de revisión desde el punto de vista de las metodologías y de las estrategias empleadas de enseñanza-aprendizaje (Gallego, 2003).

Tomando en cuenta el campo sobre el cual se dirige este estudio, se cree que este se debe dedicar principalmente a mejorar las competencias en la formación inicial del docente, en este caso del Maestro Especialista de Educación Física, basándose en el perfil que el sistema educativo actual necesita.

Esto se refiere, en este caso, al personal docente involucrado en las
Ciencias de la Educación con un campo de trabajo y estudio organizado en un ambiente institucional y que además emplea el cuerpo y el movimiento humano como el principal componente del proceso de enseñanza-aprendizaje de la Educación Física. Así, para este caso, el perfil de docente buscado es el que, según Romero y Cepero (2002), posee el conjunto de cualidades más características que debe poseer el profesional de la Educación Física y que ello le sirva de base para las funciones que desempeñe en su ámbito de actuación.

El personal docente universitario en la actualidad tiene la función de preparar al alumnado para sobrevivir y trabajar en un ambiente cambiante y, a su vez, guiarlos para que no dependan sólo de los conocimientos aprendidos, sino que también tengan la disposición de aprender nuevos contenidos de su propia experiencia, así como la capacidad de enfrentar y resolver nuevos problemas en su futura practica (Rué y de Corral, 2007).

Si se toman en cuenta los cambios que la sociedad actual exige, se puede considerar que ella se encuentra en un momento clave para que los estudiantes aprendan a desarrollar procesos cognoscitivos que deben ser aplicados a situaciones inéditas y no sólo aplicaciones del conocimiento. Es una nueva función de todo docente, porque no se enseña igual (tampoco se aprende igual) un conocimiento establecido, probado, comprobado, que una habilidad, una actitud, o lo más demandado ahora por la sociedad, una competencia (Bazdresch, 1998).

El concepto de competencia es diverso según el ángulo del cual se mire o el énfasis que se le otorgue a uno u otro elemento, pero el más generalizado y aceptado es el de "saber hacer en un contexto". El "saber hacer", lejos de entenderse como "hacer" a secas, requiere de conocimiento (teórico, práctico o teórico-práctico), afectividad, compromiso, cooperación y cumplimiento, todo lo cual se expresa en el desempeño, también de tipo teórico, práctico o teóricopráctico (Posada, 2004). 
Este trabajo pretende establecer un fundamento del conocimiento necesario y la forma de cómo podría llevarlo a la práctica el futuro docente en Educación Física. Se parte de la idea principal de que el alumnado mejore sus competencias al basarse el docente en la aplicación de estrategias y técnicas que fomenten un aprendizaje más responsable y activo, para que se pueda lograr un mejor desenvolvimiento en su experiencia profesional y alcance una mejor enseñanza de la Educación Física.

El Proyecto TUNING (Tunning Educational Structure in Europe) se presenta como uno de los principales documentos de trabajo utilizados por la comunidad universitaria en la formación inicial de estudiantes y sigue las directrices del documento marco para la integración del sistema universitario español en el Espacio Europeo de Enseñanza Superior. En este trabajo se consideran principalmente las competencias genéricas, que identifican atributos compartidos que pudieran generarse en cualquier situación. Con respecto a estas, González y Wagenaar (2003) distinguen tres tipos:

1. Competencias instrumentales: capacidades cognitivas, metodológicas, tecnológicas y lingüísticas.

2. Competencias interpersonales: capacidades individuales tales como habilidades sociales (interacción y cooperación sociales).

Competencias sistémicas: capacidades y habilidades relacionadas con sistemas globales (combinación de comprensión, sensibilidad y conocimientos; para ello es preciso adquirir previamente competencias instrumentales e interpersonales).

\section{Preguntas de investigación}

Se ha utilizado la plataforma SWAD en la asignatura de Educación Física y su
Didáctica I tomando en cuenta la facilidad de aplicarla a los alumnos del curso (se había utilizado en la asignatura en años anteriores) y, además, por la posibilidad que esta presenta de mejorar la calidad de las tareas propias del curso.

Como se dijo anteriormente en el apartado del marco teórico, la utilización de la plataforma SWAD como fórmula innovadora no implica hacer lo mismo que en las metodologías tradicionales y es un instrumento valioso que mejora tanto la enseñanza de los docentes como el aprendizaje de los estudiantes, además de mejorar la interacción entre ambos. Teniendo en cuenta esto, se ha propuesto una investigación que diera respuesta a los interrogantes de la aplicación de la Plataforma de Apoyo a la Docencia (SWAD) como una opción metodológica en la formación de los futuros maestros en Educación Física, preguntándose:

1. ¿Es la Plataforma de Apoyo a la Docencia una estrategia metodológica fundamentalmente lógica que ayuda al alumnado a desenvolverse, a adquirir las competencias y a facilitarle el proceso de aprendizaje?

2. ¿Podrán los docentes disponer de estas estrategias metodológicas para ayudar a la autonomía del alumnado y a que este mismo sea el protagonista de su propio aprendizaje?

3. ¿Favoreció la aplicación de las estrategias metodológicas el aprendizaje decompetencias generales y las específicas del programa del título de Maestro Especialista en Educación Física?

4. ¿Las diversas situaciones que se presentan en la aplicación de las estrategias metodológicas se encuentran relacionadas con el perfil profesional del Maestro Especialista en Educación Física? 


\section{Objetivos}

objetivos:

El presente estudio tiene como

1. Comprobar las valoraciones y el efecto de la aplicación de la Plataforma de Apoyo a la Docencia (SWAD) como opción metodológica en el alumnado de primer curso de la titulación de Maestro de la especialidad en Educación Física de la Universidad de Granada España.

2. Interpretar las experiencias que tiene la población estudiantil en cuanto al empleo de la Plataforma de Apoyo a la Docencia (SWAD) como una opción de estrategia metodológica y su utilidad en el desarrollo de las competencias profesionales.

\section{Metodología}

Mediante una perspectiva orientada a la práctica educativa (investigación educativa) de diseño pre-experimental (Buendía, 1992; Hernández, 1998; Hernández y Cuesta 2009), comparando dos grupos de medidas no relacionadas y utilizando una metodología mixta (cuantitativa y cualitativa) aplicada, se pretende interpretar las reacciones de los estudiantes sobre la Plataforma de Apoyo a la Docencia. Esto con el fin de orientar los procesos metodológicos que se están llevando a cabo dentro de la enseñanza universitaria para llegar, tomando decisiones y realizando cambios, a un conocimiento útil para el desarrollo de competencias que tengan, desde el punto de vista de este trabajo, una orientación hacia la inserción laboral y social y estén basadas en la clasificación de las competencias genéricas del proyecto Tuning. Además, deben ser examinadas mediante el análisis documental de las experiencias del estudiantado acerca del empleo de la Plataforma de Apoyo a la Docencia y su utilidad en el desarrollo de competencias profesionales. En este contexto, la opción de investigación complementaria de datos cuantitativos y cualitativos responde a intentar buscar una mejor comprensión del objeto de estudio de esta investigación, lo que no se lograría si se aplicara una sola metodología (Latorre y Arnal, 2003).

\section{Participantes}

El presente estudio se realizó durante el curso lectivo 2007-2008. La muestra utilizada fue de tipo intencional, así que todos los elementos de la población fueron seleccionados bajo estricto juicio del investigador tomando como muestra el grupo de estudiantes del curso de Educación Física y su Didáctica I de la titulación de Maestro Especialista de Educación Física impartida en la Facultad de Ciencias de la Educación de la Universidad de Granada (España); el curso era semestral, con asistencia obligatoria, dos veces a la semana y en el cual fueron aplicados diferentes enfoques y estrategias metodológicas (trabajos en grupo, individuales, entre otras), dentro de las cuales la Plataforma de Apoyo a la Docencia tuvo un papel preponderante en el desarrollo integral de la asignatura. La decisión de utilizar la plataforma SWAD en la asignatura de Educación Física y su Didáctica I se debió principalmente a dos razones: la primera, por la facilidad de aplicarla con el alumnado del curso (se había utilizado en la asignatura en años anteriores) y, la segunda, porque facilita los aprendizajes mediante su utilización tanto por parte del docente como del grupo de alumnos, ahorrando a su vez tiempo y mejorando la calidad de las tareas propias del curso.

Como se dijo en el apartado del marco teórico, para lograr la aplicación de la metodología de la Plataforma de Apoyo a la Docencia fue preciso extender a los estudiantes del curso la oferta de servicios 
por medio de tecnologías. Algunas de las actividades no presenciales que los estudiantes realizaron mediante el uso de la plataforma fueron:

- Lectura y estudio de los temas de la materia.

- Revisión sobre literatura específica para el desarrollo de determinados trabajos.

- Preparación y discusión de trabajos en grupo.

- Redacción del diario de campo.

- Conferencias y exposiciones on-line.

- Foros de discusión.

- Debate dirigido y discusiones.

- Resolución de problemas.

- Estudios de caso.

Cabe destacar que se trataron los datos como grupos independientes, puesto que al inicio del curso no se presentó todo el grupo de estudiantes matriculados, por lo cual se tuvo acceso a sólo 111 estudiantes y, al final del curso, lograron concluir la aplicación completa un total de 114 estudiantes.

Debido a la estructura de la asignatura y al diseño de la investigación, el total de la muestra fue dividido en dos grupos: el primero, llamado Grupo de Expectativas: (GE) estuvo constituido por un total de 111 sujetos participantes que decidieron adoptar al inicio del curso la innovación educativa que incluía la Plataforma de Apoyo a la Docencia como estrategia metodológica y expresaron mediante el instrumento utilizado sus expectativas iniciales con respecto a dicha aplicación. En cuanto al sexo de esta primera muestra, el $68 \%(\mathrm{n}=75)$ de la muestra estaba compuesta por hombres y el $32 \%$ ( $\mathrm{n}=$ 36) por mujeres, con un rango de edad entre los 17-19 años (53\%); esta disminuye conforme se aumenta el rango de edad, 20-22 (24\%), 23-25 años (15\%) y más de 26 años (8\%). El segundo, llamado Grupo de Valoraciones: (GV) estuvo constituido por un total de 114 sujetos, quienes al final del curso expresaron, mediante el instrumento utilizado, sus valoraciones finales con respecto a dicha aplicación. El 59\% (n = 67) de la muestra estuvo compuesta por hombres y el $41 \%(n=47)$ por mujeres, con un rango de edad entre los 17-19 años (47\%), disminuyendo esta conforme se aumenta el rango de edad, 20-22 (23\%), 23-25 años (21\%) y más de 26 años (9\%).

\section{Técnica o fuentes de los datos}

Se debe tomar en cuenta que para alcanzar los objetivos de investigación se utilizó una metodología mixta. Se tomó en cuenta para la parte cuantitativa la utilización de un cuestionario sobre diferentes opciones y estrategias metodológicas que incluía la metodología de la Plataforma de Apoyo a la Docencia.

El cuestionario utilizado incluía seis preguntas cerradas que permitieron recoger las manifestaciones realizadas por los estudiantes de manera no directa, facilitando así la comparación estadística. Para las alternativas de preguntas, se ha tenido en cuenta la graduación del valor del ítem para lograr codificar las alternativas de respuestas. Cada ítem se ha valorado según la escala Likert (desde $1=$ muy deficiente a $5=$ muy bueno). Para la construcción del cuestionario se acudió a la literatura especializada referida a la metodología de Plataforma de Apoyo a la Docencia y a la validación mediante expertos en docencia universitaria. De la consulta pormenorizada de un material científico actual (Adell, 1998; Celestino, Echegaray, y Guenaga, 2003; Barquín, 2004; Cañas, Ortigosa, Fernández, Anguita, Ros, y Díaz, 2004; Cañas et al. 2005; Espinosa, Jiménez, Olabe y Basogain, 2006; Cañas et al. 2007; Elgort, Smith y Toland, 2008) se extrajeron un conjunto de percepciones claves que sirvieron como base para la construcción de cada ítem del instrumento con los objetivos de la investigación (ver tabla 1).

La definición operacional de las seis variables de estudio mediante las cuales se definió la estrategia metodológica aplicada 
se realizó a partir de la revisión del concepto Plataforma De Apoyo Docente. Esta es una aplicación informática de ayuda a la enseñanza presencial que favorece la comunicación fluida entre el profesorado y los estudiantes. Como se anotó antes, por medio de ella los usuarios tienen acceso en todo momento a la información y documentación de interés para la asignatura, posibilitando la realización de diversas actividades a distancia.

Las variables dependientes y las principales descripciones de sus propósitos fueron identificadas siempre de acuerdo a los objetivos del estudio y se decidieron de la siguiente forma.

Primeramente, se acudió a la literatura especializada del campo de estudio que se refiriera a las plataformas de apoyo a la docencia (Adell, 1998; Celestino et al. 2003; Barquín, 2004; Cañas et al, 2004; Cañas et al. 2005; Espinosa et al., 2006; Cañas et al. 2007; Elgort et al. 2008). De la consulta pormenorizada del material científico recopilado, se extrajeron un conjunto de percepciones claves ajustadas al tema y que reflejaran la literatura consultada, de la cual se da cuenta en la definición de 6 diferentes ítems que reflejan el concepto y utilidad de la Plataforma de Apoyo a la Docencia (ver tabla 1).

Segundo, se verificó la validez y fiabilidad del cuestionario. Una vez redactados los ítems de cada dimensión, se comprobó si discriminaban adecuadamente efectuando un análisis calculados sobre la correlación del ítem-total con todos los demás, obteniendo correlaciones que oscilaron entre $r=0,431 y \quad r=0,509$. Esto demostró que, al estar todos los ítems por encima de $\mathrm{r}=$ 0,30 , están homogéneamente relacionados con la escala a la que pertenecen (Nurosis, 1993), atendiendo a los estadísticos descriptivos y al intervalo de confianza en el $95 \%$ de las correlaciones ( $p<0,01$ y 0,05$)$.

Tercero, para la consistencia interna (cálculo de la fiabilidad) de las preguntas del cuestionario se empleó el coeficiente alfa de Cronbach, obteniendo para los ítems un $\alpha=0,883$. Lo que demuestra que el cuestionario no sólo discrimina sobre este estudio sino que además tiene muy buena fiabilidad y consistencia interna.

Finalmente, por otro lado, para la parte cualitativa se realizó el análisis del portfolio del alumnado construido durante el curso como una herramienta de enseñanza-aprendizaje y de evaluación de los contenidos y el aprendizaje de competencias profesionales del futuro maestro especialista en Educación Física, aprovechando así su doble función y también como método para la reflexión y valoración de las opiniones (Hays, 2004). En este caso, el estudio se ha centrado en aquellas reflexiones que han efectuado los alumnos que estuvieron implicados en la experiencia de innovación docente sobre la aplicación de esta metodología educativa y que implicaran un aprendizaje más activo. Se trata en este caso de un portfolio didáctico entendido como:

Un proceso dinámico mediante el cual los docentes y/o estudiantes reúnen los datos provenientes de su trabajo y crecimiento profesional y académico respectivamente, organizados por ellos sobre la base de la reflexión, la discusión y el consenso con otros colegas y el tutor-asesor del proceso. (Lyons, N., 1999, p. 11)

\section{Análisis de los datos}

Una vez obtenidos los datos del cuestionario, estos fueron almacenados en una hoja de cálculo del programa estadístico SPSS, versión 15.0 para Windows (con licencia de la Universidad de Granada) como archivos de extensión .sav, para poder ser tratados estadísticamente con el programa y realizar los análisis oportunos.

Para el tratamiento estadístico del cuestionario se ha realizado un análisis descriptivo e inferencial. En el primer caso, se han utilizado los descriptivos básicos (media, desviación típica y distribución de frecuencias). En el segundo caso, para comparar los resultados obtenidos 
entre las expectativas iniciales (GE) y las valoraciones finales $(\mathrm{GV})$, una vez aplicada durante el curso la estrategia metodológica (SWAD) y, así, para comprobar los niveles de significación entre los grupos (GE y GV), se utilizó, debido a la ordinalidad de los datos, la prueba inferencial no paramétrica de U de Mann-Whitney (ver tabla 2).

En cuanto al análisis de los resultados cualitativos, se ha partido de la cantidad de datos en forma de texto obtenidos a partir del portafolio de estudiante desde una configuración cualitativa. Una vez obtenido los datos se ordenó toda la información en un sólo texto, al cual se le dio lectura. De esta se establecieron diversos niveles progresivos de reducción y estructuración teórica de la información mediante la construcción de las categorías y el establecimiento de los códigos de ellas una vez que fueron revisados los textos (análisis del contenido) y reformuladas las categorías previamente definidas con respecto al objeto de estudio. Esta fue una identificación de categorías relevantes para el estudio, las cuales permitieron interpretar y establecer las teorías.

De acuerdo con los objetivos de la investigación y los resultados acumulados en la base de datos, se decidió utilizar el File Maker Pro 5 para la organización y el análisis por sus características de velocidad, fiabilidad, precisión y además por su capacidad para automatizar muchas tareas repetitivas.

El sentido del análisis de datos en la investigación cualitativa consiste en reducir, categorizar, clarificar, sintetizar y comparar la información para obtener una visión lo más completa posible de la realidad objeto de estudio (Pérez, 1998). Para estos menesteres el File Maker Pro 5 fue de gran utilidad, porque permitió organizar la información de muchas formas, de modo que la información no sólo se conservara sino que se pudiera organizar y analizar y hacer comprensible su significado. El apoyo de este programa informático posibilitó la reducción de la información y su búsqueda, permitiendo escrutar registros que coincidieran con ciertos criterios o que en algunos casos excluyeran otros. Además, una vez encontrados estos conjuntos de registro, el programa permitió ordenarlos de acuerdo a lo requerido (análisis de contenido convencional).

\section{Resultados}

\section{Datos cuantitativos}

El análisis descriptivo de la estrategia metodológica estudiada (Tabla 1) presenta unas expectativas altas (media $=3,95$ ) $\mathrm{y}$ valoraciones finales igualmente altas (media $=4,31$ ). Al enfocarse en las medidas centrales del análisis descriptivo de cada uno de los ítems, se encuentra que todos suelen tener puntuaciones altas. Situándose en las expectativas, el ítem 5 "Facilitar la comunicación con otros compañeros y con el profesor de la asignatura" obtiene la mejor puntuación, con una media $=4,28$; y, en las valoraciones finales, el ítem 2 "Disponer y compartir información relevante de la asignatura", con una media $=4,46$.

Los items peor valorados ( 1 y 6 ) en las expectativas son los correspondientes a "Apoyar y orientar al alumnado en el desarrollo del aprendizaje con respecto a la asignatura" y "Aprender cuando se dispone de las posibilidades de acceso a Internet y de un ordenador personal", con una media ambos de 3.81. En el caso de las valoraciones finales, todos los ítems están puntuados por encima de 4 -muy bien valorados- por lo que todos ellos obtienen una mejora en los puntuaciones con respecto a a las expectativas iniciales.

En el gráfico 1, se puede apreciar las buenas puntuaciones de los estudiantes sobre cada uno de los ítems con los que se describe en la Plataforma de Apoyo a la Docencia. Se destaca que en todos los items se presentan mejoras en el porcentaje de frecuencias de acuerdo con la opinión de los estudiantes en las valoraciones finales, principalmente en las alternativas de respuestas de "Bastante" y "Mucho" con respecto a las expectativas iniciales. 
Tabla 1

Resultados de la estadística descriptiva según expectativas iniciales y valoraciones finales en la aplicación de la Plataforma de Apoyo a la Docencia

\begin{tabular}{|c|c|c|c|c|}
\hline \multirow{2}{*}{\multicolumn{2}{|c|}{$\begin{array}{c}\text { Plataforma de Apoyo a la Docencia } \\
\text { Ítems }\end{array}$}} & Expectativas (n: 111) & Valoraciones (n: 114) & Promedio \\
\hline & & Media (DS) & Media (DS) & Media (DS) \\
\hline 1. & $\begin{array}{l}\text { Apoyar y orientar al estudiante en el } \\
\text { desarrollo del aprendizaje con respecto } \\
\text { a la asignatura. }\end{array}$ & $3.81(0.91)$ & $4.18(0.87)$ & $4.00(0.89)$ \\
\hline & $\begin{array}{l}\text { Disponer y compartir información } \\
\text { relevante de la asignatura. }\end{array}$ & $4.05(0.95)$ & $4.46(0.81)$ & $4.26(0.88)$ \\
\hline 3. & $\begin{array}{l}\text { Tener acceso a recursos de interés prác- } \\
\text { tico para la formación académica y para } \\
\text { el futuro profesional como maestro. }\end{array}$ & $4.02(0.89)$ & $4.37(0.78)$ & $4.20(0.84)$ \\
\hline 4. & $\begin{array}{l}\text { Acercar al conocimiento y dominio } \\
\text { de las nuevas Tecnologías de la } \\
\text { Información (TIC). }\end{array}$ & $3.91(0.99)$ & $4.18(0.87)$ & $4.05(0.93)$ \\
\hline & $\begin{array}{l}\text { Facilitar la comunicación con otros com- } \\
\text { pañeros y el profesor de la asignatura. }\end{array}$ & $4.12(0.89)$ & $4.43(0.83)$ & $4.43(0.83)$ \\
\hline 6. & $\begin{array}{l}\text { Aprender cuando se dispone de las } \\
\text { posibilidades de acceso a Internet y de } \\
\text { un ordenador personal. }\end{array}$ & $3.81(1.03)$ & $4.24(0.77)$ & $4.03(0.90)$ \\
\hline & medio & $3.95(0.94)$ & $4.31(0.82)$ & $4.13(0.88)$ \\
\hline
\end{tabular}

Gráfico 1

Frecuencia porcentual de expectativas y valoración final estrategia metodológica plataforma digital

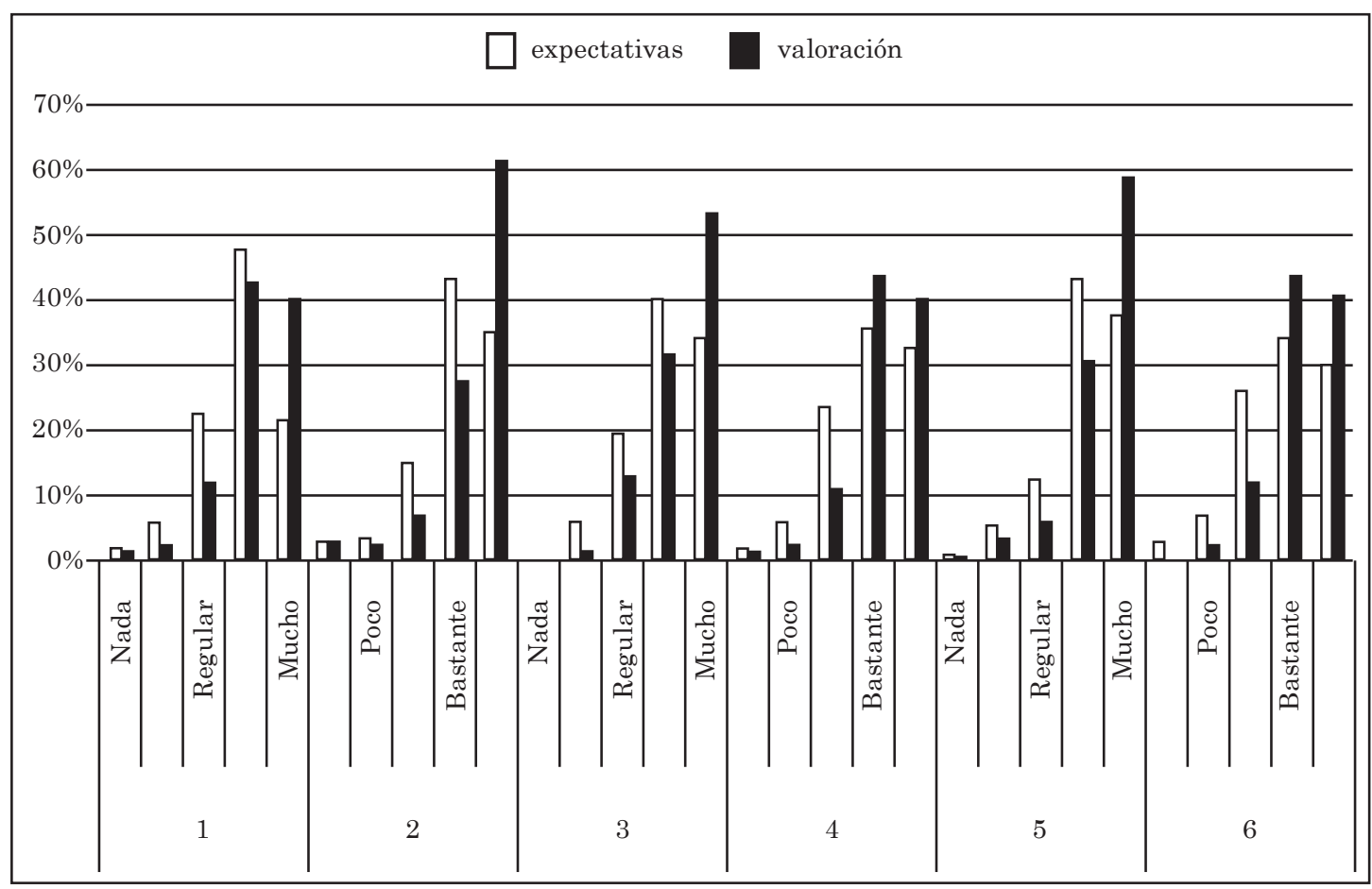


Con respecto al análisis de contraste, mediante la prueba no paramétrica de U de Mann-Whitney los resultados obtenidos indican que para los estudiantes del curso de Educación Física y su Didáctica I, luego de la aplicación de esta estrategia metodológica, existen diferencias significativas $(p<0,01$ y 0,05 ) entre las expectativas iniciales y las valoraciones finales, en todos los ítems incluidos en el análisis.

Tabla 2

Estadísticas de contraste entre expectativas y valoraciones en la aplicación de la Plataforma de Apoyo a la Docencia.

\begin{tabular}{|c|c|c|c|}
\hline $\begin{array}{c}\text { Plataforma de Apoyo a la Docencia } \\
\text { Ítems }\end{array}$ & U de Mann-Whitney & Z & Significativas \\
\hline $\begin{array}{l}\text { 1. Apoyar y orientar al estudiante en el desarrollo del } \\
\text { aprendizaje con respecto a la asignatura. }\end{array}$ & 4816,000 & $-3,316$ & 0,001 \\
\hline $\begin{array}{l}\text { 2. Disponer y compartir información relevante de la } \\
\text { asignatura. }\end{array}$ & 4572,500 & $-3,921$ & 0,000 \\
\hline $\begin{array}{l}\text { 3. Tener acceso a recursos de interés práctico para la } \\
\text { formación académica y para el futuro profesional } \\
\text { como maestro. }\end{array}$ & 4929,000 & $-3,078$ & 0,002 \\
\hline $\begin{array}{l}\text { 4. Acercar al conocimiento y dominio de las nuevas } \\
\text { Tecnologías de la Información (TIC). }\end{array}$ & 5321,500 & $-2,193$ & 0,028 \\
\hline $\begin{array}{l}\text { 5. Facilitar la comunicación con otros compañeros y } \\
\text { con el profesor de la asignatura. }\end{array}$ & 4934,500 & $-3,121$ & 0,002 \\
\hline $\begin{array}{l}\text { 6. Aprender cuando se dispone de las posibilidades de } \\
\text { acceso a Internet y de un ordenador personal. }\end{array}$ & 4884,500 & $-3,135$ & 0,002 \\
\hline
\end{tabular}

\section{Resultados de los datos cualitativos}

En el análisis de contenido del portfolio de los estudiantes, resalta la separación en unidades generales que resumen el total de los datos, unidades referidas a las competencias genéricas obtenidas por los estudiantes después de ser aplicada la estrategia metodológica; estas a la vez están representadas en diferentes categorías nombradas de la siguiente forma:

- $\quad$ Capacidad para comunicarse eficientemente de forma escrita, mediante la preparación de diferentes tipos de documentos escritos.
- Capacidad de trabajar de manera individual mediante aprendizajes activos.

- Capacidad de análisis y síntesis de diferentes documentos con el fin de actualizar conocimientos.

- Capacidad para utilizar ordenadores personales y software (programas informáticos) sencillos para efectuar tareas simples.

Para muchos de los alumnos, la aplicación de la innovación no sólo se centró en la transmisión de conocimientos, sino que también les proporcionó muchas herramientas para que construyeran sus propios aprendizajes. La aplicación de estas nuevas 
estrategias les da más autonomía y control de su propio aprendizaje:

“....El primer día de clase, en el cual se nos intentó dar una visión sobre el global de la asignatura, y para que nos hiciéramos una idea del trabajo que conllevaba la aplicación de la plataforma. A partir de este día, el trabajo corre por nuestra cuenta y la mayor o menor implicación va a depender de la que le quisiéramos dar a la asignatura también". (Sujeto $\mathrm{N}^{\circ} 70$ )

Sobre el uso de la plataforma SWAD como estrategia metodológica, el alumnado estima que se trata de una herramienta importante para el apoyo a la docencia universitaria. Principalmente, por los beneficios brindados en aspectos relacionados a una mejor comunicación con los demás grupos de compañeros y el fácil acceso a toda la información utilizada durante el curso:

\section{“...La cantidad de información que hemos tenido que manejar de la plataforma y buscar en libros, revistas, Internet, etc., nos ha ayudado para que los trabajos del curso quedaran bien". (sujeto $\mathrm{N}^{\circ} 93$ ) \\ "En ocasiones se ha producido una falta de enten- dimiento entre el profesor y gracias a utilizar la plataforma algunos compañeros me han aclarado los confusiones”. (Sujeto N ${ }^{0}$ 7)}

Sin embargo, los estudiantes que tuvieron dificultades en la utilización de la plataforma, fueron debido a los atrasos en el momento de colgar la información para que estuviera accesible a todos los del grupo:

"Los archivos deberían colgarse antes y no tan tarde que provoca que se acumule el trabajo....". (Sujeto $\left.\mathrm{N}^{\circ} 67\right)$

"También destacar que a la hora de colgar los trabajos los grupos no han cooperado mucho". (Sujeto N 12 )

Otro de los aspectos que no deja de preocupar a algunos estudiantes es el hecho de que no todos ellos tienen la misma facilidad de acceso a Internet:

"Creo que de esta manera está bien, ya que hace al alumno participe de la materia, pero veo un incon- veniente para los alumnos que no tengan Internet de forma constante. Creo que la dificultad, en gran medida, el seguimiento de las clases". (Sujeto $N^{\circ} 40$ )

\section{Discusión y conclusiones}

Destaca el alumnado como muy importante para ellos la facilidad que permite esta opción metodológica para poder comunicarse con los demás compañeros, así como el aspecto de disponer de la información más relevante de la asignatura. Por consiguiente, esta evaluación positiva muestra la disponibilidad de los estudiantes de aprender mediante técnicas de enseñanza y aprendizaje innovadoras basadas en programas informáticos, evaluaciones que concuerdan con las obtenidas por Correa (2007) durante su experiencia utilizando plataformas educativas. Gallego (2003), por su parte, en su investigación concluye que el inicio de la práctica profesional apoyada en un grupo de trabajo que se comunica mediante un foro de discusión, además de los recursos informativos que ofrece, anima a sus miembros a compartir experiencias y a colaborar ayudando a establecer buenas prácticas.

Igualmente, Romero (2008) comprobó que la Plataforma de Apoyo a la Docencia se conforma como un medio de documentación al disponer de información relevante de la asignatura, además de posibilitar compartir experiencias y como medio de comunicación entre los propios estudiantes o con el profesor. Esta proporciona autonomía y auto-aprendizaje al poder acceder a ella en cualquier momento. Igualmente, el estudiante se va habituando y adquiriendo competencias sobre las Tecnologías de la Información y la Comunicación (TIC).

En los resultados sobre el análisis de contraste para verificar si existen diferencias entre las diversas ideas que se expresan sobre esta opción metodológica, se pueden subrayar diferencias entre las expectativas y las valoraciones, comprobándose opiniones positivas y con diferencias significativas del 
cuestionario final respecto al cuestionario inicial $(p \leq 0.05)$ en todos los ítems que definen esta estrategia; sin embargo, estas aseveraciones no han sido comprobadas por otros autores, presumiendo que este tipo de análisis no ha sido realizado de igual manera en otros estudios relacionados.

Respecto a la valoración que realizan el grupo de alumnos sobre el apoyo $y$ orientación que reciben sobre la asignatura con el uso de la plataforma, se ha podido comprobar el efecto que produce cuando se ha utilizado en el desarrollo de la asignatura. Durante una observación del aprendizaje basado en la web del alumnado y su entorno, Marty, Heraud, Carron y France (2007) demostraron para su comprensión que la disposición de este tipo enseñanza y su entendimiento en algunos casos puede conducir a mejorar la situación de aprendizaje entre los estudiantes. Concuerdan estos resultados con los obtenidos con los estudiantes del presente estudio, quienes consideran que la Plataforma de Apoyo a la Docencia les afirma y orienta en el desarrollo de los aprendizajes con respecto a los contenidos de la asignatura.

Un segundo aspecto muestra significación en la valoración final de los estudiantes sobre las posibilidades que ofrece la plataforma de disponer y compartir información relevante de la asignatura. Coinciden estos resultados con los obtenidos por Kakehi, Kura, Kusaka, Fujino, Fujimoto y Takahashi (2001), quienes demostraron que este tipo de aprendizaje permite a los estudiantes aprender de una manera más efectiva y significativa, ofreciéndoles un ambiente colaborativo que tiene como base el conocimiento que cada estudiante genera y la posibilidad de tener acceso a comentarios, opiniones e informaciones también generados por otros, incluso de manera virtual, mediante la utilización acertada de esta herramienta electrónica.

También se aprecian las diferencias de la valoración con respecto a los posicionamientosiniciales de los estudiantes sobre poder acceder a los recursos de intereses prácticos para una formación académica que les asegure un buen desarrollo profesional. Al respecto, Martínez, Mileva, Tzanova, Castro, Bastiaen, Stoyanov et al. (2004) apoyan la utilización de plataformas educativas en los cursos universitarios por sus posibilidades para la realización de prácticas simuladas, tareas, proyectos, junto con las posibilidades de interactividad entre alumnos. Igualmente, argumentan que siempre está disponible en el momento en que se precisa, y ofrece la posibilidad de adaptarse a las diferentes necesidades y niveles de formación de los usuarios.

Del mismo modo se aprecian diferencias entre las valoraciones sobre las expectativas, porque para el alumnado es primordial adherirse al conocimiento y dominio de las nuevas Tecnologías de la Información (TIC), debido a que les permite el acceso a información necesaria en cualquier lugar. Además, esas diferencias son evidentes cuando han podido comprobar que les ha permitido comunicarse con los demás compañeros para intercambiar ideas y materiales. Barquín (2004) agrega al respecto que la filosofía a recurrir en los centros que aplican nuevas tecnologías de información no debe de ir encaminada a "aprender informática", sino a "usar la informática para aprender". Otro de los indicadores sobre el cual los resultados muestran una valoración final positiva es la facilidad de comunicación con otros compañeros que provee la Plataforma de Apoyo a la Docencia. La misma percepción muestran los estudiantes en otras investigaciones realizadas (Correa, 2007; Elgort et al., 2008), que valoraron de forma positiva el uso de las plataformas educativas por considerarlas una buena forma de encontrar información y además útil para organizar y compartir el conocimiento con los demás compañeros.

Finalmente, el último aspecto de esta estrategia metodológica que también muestra diferencia significativa en sus valoraciones finales con respecto a las expectativas es el aprender cuando 
se dispone de la posibilidad de nuevas tecnologías de información. Sobre este punto aclaran Celestino et al., (2003) que las TIC no implican por sí solas una mejora en la calidad de la formación, sino que esto se da según la forma de utilizarlas para que contribuyan efectivamente durante el proceso de aprendizaje; sólo bajo este concepto se les puede llegar a considerar una verdadera herramienta de apoyo a la docencia.

El poder poner en práctica estas nuevas tecnologías de la información y comunicación (TIC) para el desarrollo de aprendizajes se clasifica como una de las competencias genéricas necesarias de adquirir por los actuales estudiantes en formación. Este tipo de competencias, dentro del proyecto TUNING (González et al., 2003), se encuadran dentro de las competencias instrumentales y se denominan habilidades básicas de manejo de ordenador y se corresponden con las destrezas tecnológicas relacionadas con el uso de maquinaria, destrezas de computación y gerencia de la información.

De acuerdo a lo exteriorizado por el alumnado, la utilización de la plataforma de apoyo docente SWAD le ha beneficiado, principalmente para gestionar y estructurar la asignatura (por temas o semanas) y para la organización de materiales didácticos (archivos de texto, video). Esto es similar a los beneficios que ha encontrado Correa (2007) en sus trabajos de investigación. Paralelamente, los estudiantes coinciden en que, aparte de facilitar la comunicación con los compañeros, la plataforma facilita el acceso a la información. Elgort et al. (2008) confirman estos resultados encontrados en cuanto a que en las plataformas educativas los estudiantes encuentran información útil para organizar conocimientos y compartirla con los demás compañeros.

Algunas consideraciones negativas sobre el uso de la Plataforma de Apoyo a la Docencia van dirigidas hacia la posibilidad que esta presenta para la realización de tutorías no presenciales, a pesar de que esta metodología tiene la potencialidad de permitir un contacto continuado e inmediato entre el docente y el alumno. Aunque se dé una creciente importancia sobre su uso en la actualidad, los estudiantes no la ven como una buena opción metodológica. En este sentido, Pérez (2006) encuentra el inconveniente de la poca profundización que suele desarrollarse en la comunicación vía electrónica.

Actualmente, se está reflejando bastante la aplicación de las TIC en los procesos de enseñanza y aprendizaje, tomando los entornos virtuales como hechos educativos que facilitan el acceso a la información, aunque esto no significa que siempre van a facilitar el acceso al conocimiento. En líneas generales los alumnos juzgan que la plataforma SWAD es un buen apoyo si se utiliza para generar oportunidades de aprendizaje y no simplemente para proveer a los estudiantes de información.

\section{Conclusiones}

Las comprobaciones y el efecto de las valoraciones sobre la aplicación de la Plataforma de Apoyo a la Docencia en alumnos de primer curso de la titulación del Maestro especialista en Educación Física ha representado una forma alternativa y permanente de comunicación con el docente y los demás grupos de compañeros, proporcionando a su vez información relevante de la asignatura.

La dimensión interpretativa sobre las experiencias del alumnado relacionadas con el empleo de la Plataforma de Apoyo a la Docencia y su utilidad para el desarrollo de competencias engloba aspectos relacionados con las satisfacciones y dificultades vertientes del aprendizaje. Con respecto a ella, se plantea la siguiente conclusión relativa a las valoraciones de los alumnos. La innovación docente aplicada a los estudiantes ha supuesto una cierta dificultad para poder llevarla a cabo, suponiendo un gran esfuerzo y 
dedicación de tiempo. La intensidad y ritmo de este tipo de trabajo, unido a la abundancia y dispersión de tareas que tienen los estudiantes universitarios, hace que la aplicación de la Plataforma de Apoyo a la Docencia sea exigente pero, al mismo tiempo, gratificante por sus implicaciones y consecuencias.

\section{Referencias bibliográficas}

Adell, J. (1998). Redes y Educación. En J. de Pablos y J. Jiménez (Eds.), Nuevas tecnologías. Comunicación audiovisual y educación. (pp. 177211). Barcelona: Cedecs Editorial.

Barquín, J. (2004). La implantación de las tecnologías de la información en la sociedad y en los centros educativos públicos de la comunidad de Andalucía. Revista Iberoamericana de educación, 36, 155-174.

Bazdresch, M. (1998). Las competencias en la formación docente. Revista de Educación Nueva Época, 6, 61-69.

Benito, A. y Cruz, A. (2005). Nuevas claves para la docencia universitaria. Madrid: Nancea.

Bolívar, A. (2006): "Proyecto de Excelencia Universitaria de la Junta de Andalucía HUM-1346: Conocimiento y Competencia Profesional Docente”. Recuperado el 27 de octubre, 2008 de http://www.ugr.es/ didlen/proyectos/ Proyecto_excelencia_Pilar.pdf

Buendía, L. (1992). El método experimental: Diseños de investigación. En P. Colás y L. Buendía. Investigación Educativa (pp. 109-153). Sevilla: Alfar.

Cañas, A., Calandria, D. J., Ortigosa, E. M., Ros, E., y Díaz, A. F. (2007). SWAD:
Web System for Education Support. En B. Fernández-Manjón, J. M. Sánchez-Pérez, J. A., Gómez-Pulido, M. A., Vega Rodríguez, y J. BravoRodríguez (Eds.), Computers and Education: E-learning, From Theory to Practice. Editorial Springers.

Cañas, A., Ortigosa, E. M., Fernández, F. J., Anguita, M., Ros, E. y Díaz, A. F. (2004, Outubro). Plataforma de teleformación SWAD. Actas da Conferência IADIS Ibero-Americana WWW/Internet 2004, 89-96. Madrid, España.

Cañas, A., Ortigosa, E. M., y Aragón, Y. (2005, marzo). La plataforma SWAD como recurso docente para la innovación educativa. Congreso internacional sobre el profesorado ante el reto de las nuevas tecnologías en la sociedad del conocimiento. Recuperado el 16 de mayo, 2009 de http://www. granhotelstgo.cu/evento/educacionadistancia/univgranada $2005 /$ Programa/04/4/12.pdf

Celestino, A., Echegaray, O., y Guenaga, G. (2003). Integración de las TIC en la educación superior. Pixel-Bit. Revista de medios y Educación, 021, 21-28.

Centro de Innovación Docente para las Universidades Andaluzas [CIDUA]. (2005). Informe sobre Innovación en la Docencia en las Universidades Andaluzas. Sevilla: Dirección General de Universidades, Secretaria de Universidades, Investigación y Tecnología. Consejería de Innovación Ciencia y Empresa de la Junta de Andalucía.

Correa, C. (2007). Impactos del aprendizaje autónomo en el diseño curricular y didáctico dentro de la práctica docente. Seminario internacional $R E D-U$. Extraído 26 Diciembre, 2008 
de http://revistas.um.es/actas-redu/ article/view/434/405

Elgort, I., Smith, A. G., y Toland, J. (2008). Is Wiki an effective platform for group course work?. AJET:Australasian Journal of Educational Technology, 24(2), 195-210.

Espinosa, J. K., Jiménez, J., Olabe, M., y Basogain, Y. X. (2006). Innovación docente para el desarrollo de competencias en el EEES. [Electronic Version]. Tecnologías aplicadas a la enseñanza de la Electrónica. Extraído 10 Febrero, 2009 de http://campus. usal.es/ ofeees/ARTICULOS/p216. $p d f$

Esteban, M., y Sáez, J. (2008, Setiembre 15). Las profesiones, las competencias y el mercado. Red U. Revista de Docencia Universitaria. Número Monográfico II, 1-16. Recuperado el 09 de enero, 2009 en http://www. red-u.org/

Fernández, E., Mir, C., y Pablo-Martí, F. (2007). Innovación docente en la enseñanza universitaria: un factor diferenciador. En J. C. Ayala Calvo (Ed.), Conocimiento, innovación y emprendedores: camino al futuro (pp. 44-55). Editorial FEDRA.

Gallego, M. J. (2003). Intervenciones formativas basadas en www para guiar el inicio de la práctica profesional de los docentes. Revista Iberoamericana de Educación. 33, 111-131.

González, J. y Wagenaar, R. (2003). Tunning Educational Structure in Europe. Bilbao: Universidad de Deusto.

Hays, R. B. (2004). Reflecting on learning portfolios [Reflexión sobre los portafolios de aprendizaje]. Medical Education, 38(8), 801-803.
Hernández，F. (1998). Conceptualización del proceso de la investigación educativa. En L. Buendía, P. Colás y F. Hernández. Métodos de Investigación en Psicopedagogía (pp. 1-60). Madrid: Mc Graw Hill.

Hernández, F. y Cuesta, J. D. (2009). Métodos cuantitativos de investigación. En P. Colás, L. Buendía y F. Hernández. Competencias científicas para la realización de una tesis doctoral (pp. 63-96). Barcelona: Davinci.

Kakehi, N., Kura, T., Kusaka, R., Fujino, M., Fujimoto, T., y Takahashi, T. (2001). Development of eTrip System: Collaborative Learning Platform for a Field Trip.

Latorre, A., y Arnal, J. (2003). Bases Metodológicas de la Investigación Educativa. Barcelona España: Ediciones Experiencia.

Lyons, N. (1999). El uso del portafolio para el aprendizaje y la evaluación. Buenos Aires Amorrortu.

Martínez, C., Mileva, N., Tzanova, S., Castro Gil, M. A., Bastiaen, T., Stoyanov, S., et al. (2004). Principales resultados de la aplicación de la plataforma educativa ipss_ee en diferentes universidades europeas. VIRTUAL EDUCA. $V$ Encuentro Internacional sobre Educación, Capacitación Profesional y Tecnologías de la Información. III Conferencia Iberoamericana de Rectores. Extraído 12 Marzo, 2008 de www.virtualeduca.org/encuentros/ barcelona2004/es/actas/6/1.6.2.doc

Marty, J., Heraud, J., Carron, T., y France, L. (2007). Matching the Performed Activity on an Educational Platform with a Recommended Pedagogical Scenario: A Multi-Source Approach 
[Coincidencia de la actividad desarrollada en una plataforma educativa con un Escenario Recomendado Pedagógica: un Enfoque MultiSource]. Journal of Interactive Learning Research, 18(2), 267-283.

Ministerio de Educación y Ciencia en España [MEC]. (2006). Propuestas para la renovación de las metodologias educativas en la universidad. Madrid: Secretaria General Técnica. Subdirección General de Información y Publicaciones.

Nurosis, M. (1993). SPSS. Statistical data analysis. New York: SPSS Inc.

Pérez, A. (2006). Tutorías. En M. de Miguel (Ed.). Metodologías de enseñanza y aprendizaje para el desarrollo de competencias (pp. 133-167). Madrid: Alianza.

Pérez, G. (1998). Investigación cualitativa. Retos e interrogantes II técnicas y análisis de datos (2 $2^{\mathrm{a}}$ ed.). Madrid: La Muralla.

Posada, R. (2004). Formación superior basada en competencias, interdisciplinariedad y trabajo autónomo del estudiante. Revista Iberoamericana de Educación 34(4), 1-33. Consultado el 11 de abril, 2009 de http://www. rieoei.org/edu_sup22.htm o en formato PDF http://www.rieoei.org/ deloslectores/648Posada.PDF

Romero, C. (2008). Un desarrollo metodológico en la titulación de maestro en educación física: un acercamiento a los nuevos enfoques de las enseñanzas universitarias. En R. Roig y J. E. Blasco (Coord.). Investigación e innovación en el conocimiento educativo actual (pp. 403-426). Alcoy (Alicante): Marfil.

Romero, C. y Cepero, M. (2002). Bases teóricas para la formación del maestro especialista en Educación Física. Granada: Grupo Editorial Universitario.

Romero, C., y Salicetti, A. (2009). La contribución del trabajo grupal de los estudiantes como estrategia docente en la formación del maestro especialista en educación física. Revista iberoamericana de educación, 49(8). Recuperado el 23 de julio, 2009 de http://www.rieoei. org/deloslectores/2952Cerezo.pdf

Rué, J., y de Corral, I. (2007, septiembre). Significados de la "Formación docente" en las universidades españolas en el marco del EEES. Red U. Revista de Docencia Universitaria. Año I. Número 2. Recuperado el 11 de abril, 2009 de http://www.red-u.org/img/ anuncios/2.htm

Sánchez, F. (2007, diciembre). Eduagora. Una visión colaborativa de las TIC en la educación. Revista DIM: Didáctica, Innovación y Multimedia, Año 3, 10, 1-7.

Shulman, L. S. (2004). Teaching as community property: Essays on higher education. San Francisco: Jossey-Bass. 